\title{
Conhecimento e habilidades de estudantes de medicina em prevenção de doenças crônicas: uso do exame clínico objetivo estruturado como ferramenta de avaliação
}

\author{
Knowledge and skills of medicine students in prevention of Chronic Diseases: use of \\ Objective Structured Clinical Examination as an evaluation tool
}

Conocimientos y habilidades de estudiantes de medicina en la prevención de Enfermedades Crónicas: uso del examen clínico estructurado objetivo como herramienta de evaluación

Edienny Augusta Viana Santos-Lobato ${ }^{1 *}$, Juliana Leão de Souza Duque ${ }^{1}$, Nelson Machado da Silva de Lima ${ }^{1}$, Milena Coelho Fernandes Caldato'.

\section{RESUMO}

Objetivo: Avaliar as habilidades de estudantes de medicina quanto a orientação de medidas para prevenção de Doenças Crônicas. Relato da experiência: Foi realizado um estudo quantitativo, observacional e transversal, com alunos do quinto semestre do curso de medicina do Universidade do Estado do Pará. Os estudantes participaram de uma estação de mini-OSCE, onde deveriam identificar fatores de risco para doença crônica, orientando médicas preventivas e elaborando plano de metas para o retorno. Vinte e quatro alunos foram avaliados quanto às atitudes, conhecimentos e habilidades em prevenção de doenças crônicas. A nota média obtida pelos alunos foi de 5,81, sendo constituída por avaliação de atitudes, conhecimentos e habilidades. Houve lacunas no reconhecimento da obesidade como fator de risco para doenças crônicas, sendo apontada por somente $45,8 \%$ dos estudantes. Na avaliação de habilidades os avaliados apresentaram dificuldades no direcionamento de uma orientação alimentar baseada em evidências, com inclusão de alimentos saudáveis. Também foi observado que menos da metade dos estudantes orientaram sobre a prática de exercício físico estruturado ou elaboraram plano de metas para reavaliação do paciente atendido. Considerações finais: há necessidade de integração e aprimoramento curricular em habilidades clínicas direcionadas ao manejo contínuo de pacientes com fatores de risco para doenças crônicas.

Palavras-Chave: Habilidades clínicas, Promoção da saúde, Doença crônica.

\begin{abstract}
Objective: To evaluate the skills of medical students regarding the orientation of measures for the prevention of Chronic Diseases. Experience report: A quantitative, observational and cross-sectional study was conducted with students of the fifth semester of the medical school of the State University of Pará. Students participated in a mini-OSCE station, where they should identify risk factors for chronic disease, advising preventive medicine and developing a goal plan for return. Twenty-four students were assessed regarding attitudes, knowledge of chronic disease prevention skills. The average grade obtained by the students was 5.81 , consisting of assessment of attitudes, knowledge and skills. There were gaps in the recognition of obesity as a risk factor for chronic diseases, being pointed out by only $45.8 \%$ of the students. In the assessment of skills, the subjects presented difficulties in directing an evidence-based dietary orientation, including healthy foods. It was also observed that less than half of the students advised on the practice of structured physical
\end{abstract}

1 Universidade do Estado do Pará, Belém-PA. *E-mail: santos-lobato@hotmail.com 
exercise or elaborated goals plan for reevaluation of the treated patient. Considerations: There is a need for integration and curriculum improvement in clinical skills directed to the continuous management of patients with risk factors for chronic diseases.

Key words: Clinical skills, Health promotion, Chronic disease.

\section{RESUMEN}

Objetivo: Evaluar las habilidades de los estudiantes de medicina con respecto a la orientación de las medidas para la prevención de enfermedades crónicas. Informe de Experiencia: Se realizó un estudio cuantitativo, observacional y transversal con estudiantes del quinto semestre de la escuela de medicina de la Universidad Estatal de Pará. Los estudiantes participaron en una estación mini-OSCE, donde deberían identificar los factores de riesgo de enfermedad crónica, aconsejando medicina preventiva y desarrollar un plan de objetivos para el retorno. Veinticuatro estudiantes fueron evaluados con respecto a actitudes, conocimiento y habilidades de prevención de enfermedades crónicas. La calificación promedio obtenida fue de 5.81. Hubo lagunas en el reconocimiento de la obesidad como factor de riesgo para enfermedades crónicas. En la evaluación de las habilidades os sujetos presentaron dificultades para dirigir una orientación dietética basada en la evidencia, incluyendo los alimentos saludables. También se observó que menos de la mitad de los estudiantes asesoraron sobre la práctica de ejercicio físico estructurado o plan de metas elaborado para la reevaluación del paciente tratado. Consideraciones finales: Existe una necesidad de integración y mejora del plan de estudios en las habilidades clínicas dirigidas al tratamiento continuo de pacientes con factores de riesgo de enfermedades crónicas.

Palabras clave: Habilidades clínicas, Promoción de la salud, Enfermedades crónicas.

\section{INTRODUÇÃO}

Desde o início do século, o Brasil presencia uma acelerada transição epidemiológica e demográfica, onde Doenças Crônicas não Transmissíveis (DCNT) se tornaram mais prevalentes e representam uma importante prioridade de saúde no Brasil (MENDES EV, et al., 2012). Estima-se que mais de 45\% da população adulta do Brasil seja portadora de alguma doença crônica, as quais são representadas principalmente por doenças cardiovasculares, respiratórias, renais, neoplasias e diabetes (MALTA DC, et al., 2017).

Essas condições têm apresentado um constante crescimento dentre as causas totais de mortalidade, correspondendo a taxas acima de $70 \%$ na proporção de óbitos anuais do país (MENDES EV, 2012). A mortalidade, no entanto, pode não refletir a carga total representada por DCNT, as quais contribuem também com o impacto negativo na qualidade de vida do indivíduo, limitação funcional devido à perda da mobilidade, aumento no número de internações hospitalares e, inclusive, impactam na própria economia do país, uma vez que geram absenteísmo, aposentadorias e redução da população economicamente ativa (MINISTÉRIO DA SAÚDE, 2013; BOCCOLINI MM, et al., 2017).

Intervenções focadas nos hábitos de vida e comportamentos dos pacientes provaram-se eficazes. Essas intervenções incluem aconselhamento pelo profissional de saúde, onde o papel do médico é vital. Os resultados positivos de orientações nas consultas médicas com relação a hábitos de vida saudáveis, está intimamente ligado à motivação e domínio do conhecimento por parte do médico. Profissionais de saúde que receberam treinamento em medicina preventiva costumam adotar para si hábitos mais saudáveis e apresentam melhor desenvoltura no aconselhamento para a prevenção e manejo de DCNT. Adiante, nota-se que as orientações são mais eficazes quando o paciente enxerga o médico como um modelo das mensagens por ele transmitidas (ALBA LH et al., 2015).

Inadvertidamente, em alguns casos a graduação médica, não são ressaltadas as diferenças que existem em relação aos cuidados de doenças agudas e doenças crônicas. Entretanto, é fundamental reconhecer que os conhecimentos, habilidades e atitudes voltados para enfermidades agudas não são diretamente aplicáveis 
às crônicas (HUGHES C, 2002). O quadro agudo possui como ênfase a doença no paciente e a importância do diagnóstico e tratamento terapêutico adequados; por outro lado, a condição crônica enfatiza o paciente com a doença e a relevância está na avaliação e manejo clínico conduzido em etapas em um planejamento longitudinal (NAIR BR e FINUCANE PM, 2003).

A educação em saúde requer novas competências, o que significa que os estudantes de Medicina devem estar preparados para a assistência de pacientes com DCNT, o que inclui o planejamento longitudinal, mudança de comportamento, aconselhamento e promoção do autocuidado (REMUS KE, 2016). É necessário enfatizar, portanto, que educação em medicina preventiva é uma intervenção imprescindível e possui como objetivo facilitar o aprendizado de futuros médicos sobre como aconselhar seus pacientes, de modo eficaz, quanto à adoção e manutenção de hábitos saudáveis (POLAK R, et al., 2015).

O Exame Clínico Objetivo Estruturado (OSCE) é uma ferramenta de avaliação validada para uso em educação médica, apresentada inicialmente por Ronald Harden, que objetiva avaliar as habilidades práticas de estudantes em um ambiente clínico simulado. Esta metodologia avalia de forma estruturada, utilizando-se uma lista de checagem de tarefas, no qual o estudante deve desempenhar corretamente cada item avaliado durante um tempo pré-determinado (BERNARD AW, et al., 2017).

O OSCE costuma ser utilizado em Educação Médica para a avaliação final do eixo de habilidades clínicas priorizando o atendimento simulado de casos que envolvem doenças agudas, o que inclui execução de anamnese, exame físico, raciocínio clínico ou plano terapêutico. Não há, no entanto, informações sobre o uso do método para a avaliação de competências clínicas para o atendimento de pacientes com doenças crônicas, nos quais a conduta envolve prioritariamente orientações sobre mudanças de estilo de vida. Deste modo, este estudo objetivou avaliar os conhecimentos e habilidades de estudantes de medicina em uma estação de consulta médica simulada, onde os mesmos deveriam orientar medidas de prevenção de Doenças Crônicas para um paciente-ator em atendimento.

\section{RELATO DE EXPERIÊNCIA}

Foi realizado um estudo quantitativo, observacional e transversal, objetivando avaliar as habilidades e conhecimentos em prevenção de Doenças Crônicas por estudantes de Medicina. A pesquisa foi efetuada no Campus de Ciências Biológicas e da Saúde (CCBS) da Universidade do Estado do Pará (UEPA) no período de agosto de 2017 a dezembro de 2018. Os participantes da pesquisa foram estudantes do quinto semestre do Curso de Medicina. A Coordenação do Curso de Medicina e o Comitê de Ética em Pesquisa da UEPA aprovaram o estudo (CAAE: 68991717.0.0000.5174) e todos os participantes consentiram sua participação através da assinatura do Termo de Consentimento Livre e Esclarecido.

Os estudantes participaram de uma estação de mini-OSCE onde foram avaliados através de um checklist quanto às atitudes (por exemplo: "apresentou-se e cumprimentou o paciente"), conhecimentos (por exemplo: "reconhece os fatores de risco para doença renal cônica que o paciente do caso clínico apresenta") e habilidades (por exemplo: esclarece sobre dieta mediterrânea). As atitudes, conhecimentos e habilidades avaliados agrupavam pontuação mínima de 0 e máxima de 10 .

Um total de 24 alunos do quinto semestre do Curso de Medicina foram convidados para a participação de uma estação de mini-OSCE com paciente-ator após o término do módulo de habilidades clínicas em doenças renais, na metade do semestre. Nesta avaliação, os alunos deveriam identificar fatores de risco para doença renal crônica, orientar medidas preventivas e elaborar plano de metas para o retorno.

A nota média obtida pelos alunos foi 5,81, sendo constituída em $10 \%$ por avaliação de atitudes, $25 \%$ sobre conhecimentos e $65 \%$ por habilidades.

$\mathrm{Na}$ avaliação de atitudes os alunos apresentaram bom desempenho, onde todos os alunos utilizaram linguagem adequada ao atendimento, evitando o uso de jargão e a maioria dos estudantes (83.3\%) se apresentou e cumprimentou o paciente-ator (Figura 1). 
Figura 1 - Avaliação de atitudes, conhecimentos e habilidades de estudantes voluntários do $5^{\circ}$ semestre do Curso de Medicina sobre prevenção de doenças crônicas.

\section{Utiliza a linguagem apropriada ao entendimento do paciente}

\section{Apresentar-se e cumprimentar o paciente \\ Reconheceu os seguintes fatores de risco modificáveis para doença renal crônica: \\ Obesidade}

Glicemia elevada

Pressăo arterial elevada

Informa sobre necessidade de adequação alimentar

Orienta sobre restriçāo no consumo de alimentos processados

Orienta sobre alimentos que devem ser incluidos na alimentação

Fornece orientaçăo sobre dieta mediterrânea

Informa sobre a necessidade de realizaçăo de exercicio físico

Reconhece a diferença entre atividade física e exercício fisico

\section{Explica sobre a realizaçăo de exercício} físico estruturado

Reconhece que exames cardiovasculares devem ser realizados na presença de sinais e sintomas

Orienta retorno com plano de açōes ou metas

$$
0 \%
$$

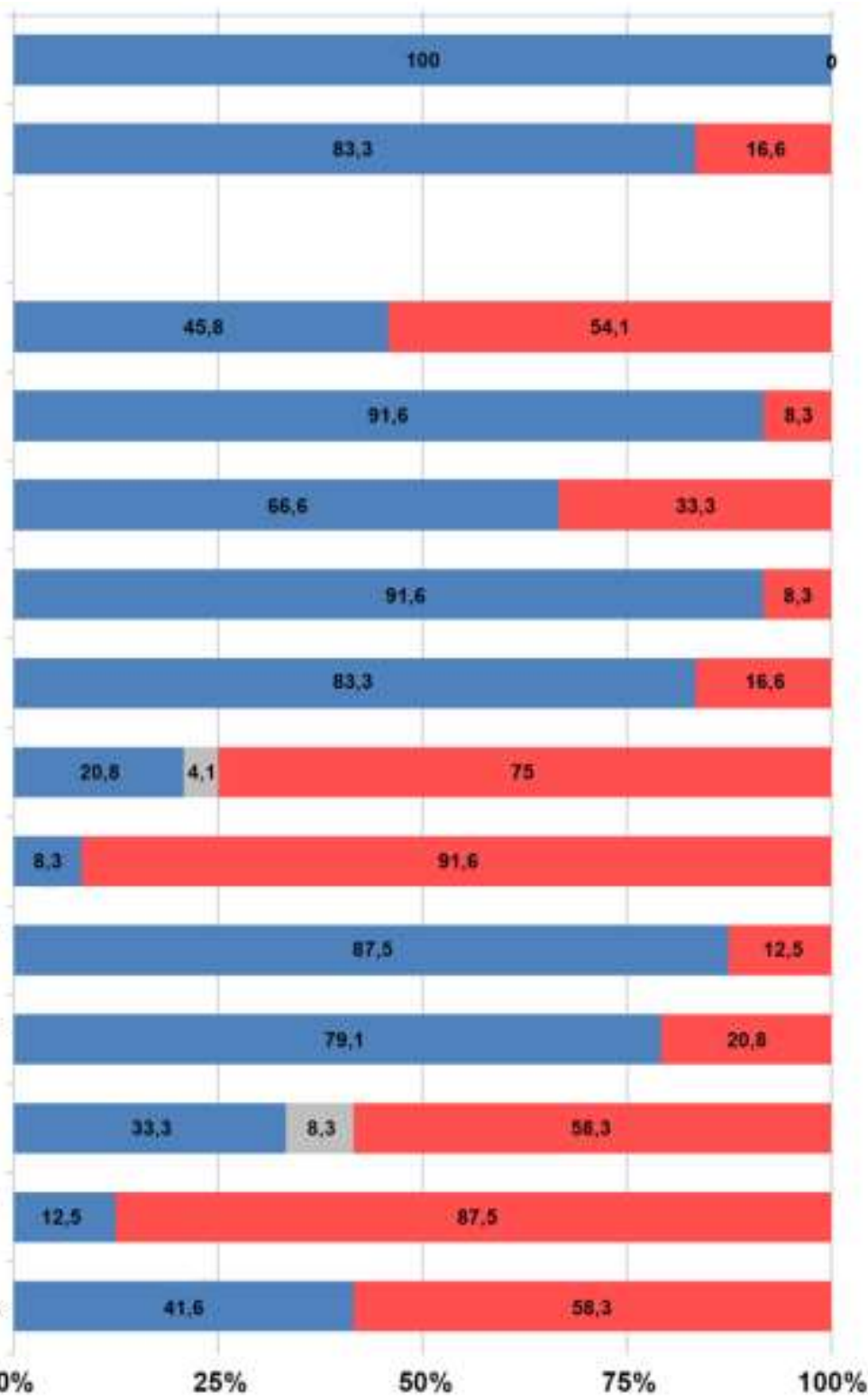

Fonte: Santos-Lobato, EAV et al., 2019. Protocolo de estação de mini-OSCE.

$\mathrm{Na}$ análise de conhecimentos os estudantes deveriam reconhecer no caso clínico exposto quais os principais fatores de risco para o desenvolvimento de doenças renais crônicas. A glicemia elevada foi a condição clínica mais frequentemente identificada (91.6\%), porém menos da metade dos estudantes (45.8\%) reconheceu a obesidade como um fator de risco.

$\mathrm{Na}$ avaliação de habilidades os alunos foram avaliados quanto às orientações direcionadas sobre alimentação, exercício físico e estabelecimento de planos de ações com metas para serem cumpridas até o atendimento de retorno. Uma frequência de $91.6 \%$ dos estudantes informou sobre a necessidade de adequar a alimentação, e a maioria dos alunos orientaram sobre a limitação no consumo de alimentos processados (83.3\%). No entanto, somente $8.3 \%$ dos avaliados conheciam a dieta mediterrânea utilizada como exemplo e apenas $20.8 \%$ citaram os alimentos que deveriam ser incluídos na alimentação do paciente.

Dentre os principais alimentos mencionados como processados, os quais deveriam ser limitados da alimentação, incluiu-se açúcar, refrigerantes, doces e pães. Metade dos alunos citou limitação no consumo de carboidratos e proteínas, sem, no entanto, fornecer informações objetivas sobre o assunto. 
Em $87.5 \%$ das avaliações, os estudantes informaram sobre a necessidade de acrescentar exercícios físicos ao tratamento preventivo e $79.1 \%$ responderam que a atividade de caminhar para o trabalho, utilizada como exemplo, não era suficiente para ser considerada como exercício físico. No entanto, somente 33.3\% orientaram o paciente sobre a realização de exercício físico estruturado e $87.5 \%$ dos estudantes confirmaram que antes de iniciar o exercício físico, o paciente precisaria realizar exames de avaliação cardiovascular. Apesar de todos os estudantes serem direcionados para o fornecimento de orientações ao paciente sobre 0 retorno, somente $41.6 \%$ estabeleceram algum plano de ações ou metas.

\section{DISCUSSÃO}

$\mathrm{Na}$ avaliação de atitudes, conhecimentos e habilidades de estudantes do quinto semestre na estação de mini-OSCE, foi observado que apesar de reconhecerem a importância de fornecer orientações alimentares para a prevenção de doenças, a maioria dos estudantes apresentou dificuldades no direcionamento desta orientação. Poucos alunos informaram sobre a necessidade de introdução de alimentos saudáveis na alimentação e a minoria reconheceu ou soube caracterizar a dieta mediterrânea. Corroborando com estes achados, Eisenberg DM e Burgess JD (2015) enfatizam sobre o desenvolvimento de instrumentos em avaliação clínica, como OSCE, para avaliações práticas relacionadas ao aconselhamento sobre estilo de vida.

Sierpina VS, et al., (2016) sugerem que educação nutricional na escola de Medicina deve capacitar os futuros médicos em doze competências mínimas, dentre as quais: conduzir uma história dietética, aprender sobre o preparo dos próprios alimentos, discutir e capacitar os pacientes na compra e preparação de uma dieta saudável, avaliar e demonstrar o conhecimento de dietas baseadas em evidências, tais como dieta mediterrânea e DASH (Dietary Approaches to Stop Hypertension), trabalhar em equipe multiprofissional, aprender e participar de entrevistas motivacionais, conectar os pacientes a recursos existentes para uma alimentação saudável, identificar pacientes que necessitem de terapia comportamental e descrever o papel de alimentos e nutrientes contrastada ao de suplementos dietéticos específicos.

De forma similar, houve dificuldades em relação à orientação sobre práticas de atividade física, onde a maior frequência de orientações foi superficial e pouco direcionada para o exercício físico estruturado, com orientações baseadas em evidências. De modo adicional, a identificação de temas sobre exercício físico no Projeto Pedagógico do Curso de Medicina foi escassa, com abordagem apenas teórica durante os semestres iniciais do curso. Tradicionalmente, não há educação formal ou experiências práticas sobre exercício físico nos Cursos de Medicina e mesmo quando este tema está inserido no currículo, foca-se tão somente em aspectos de fisiologia, com menor atenção ao seu papel na prevenção de doenças e habilidades em aconselhamento (CARDINAL BJ, et al., 2015).

Outros estudos também identificaram a escassez de prescrições de exercício físico por médicos na prática clínica regular e este dado esteve relacionado à falta de conhecimento e falta de confiança em aconselhamento, com dificuldade de incorporar a prescrição de exercício físico em seus atendimentos de pacientes (O'BRIEN MW, et al., 2017).

A minoria dos alunos também incorporou o estabelecimento de metas na orientação de retorno do paciente em programação de mudança de estilo de vida. A elaboração de plano de metas é uma estratégia importante na promoção do autocuidado e está associada a melhora dos resultados em saúde, devendo ser incorporada no atendimento de pacientes em acompanhamento longitudinal (SAUCIER AN, et al., 2017).

Acredita-se que a educação médica voltada para a formação de estudantes em medicina preventiva poderá criar uma geração de médicos com conhecimentos, habilidades e ferramentas para melhorar e manter comportamentos saudáveis para seus pacientes (POLAK R, et al., 2017).

Adicionalmente, essas mudanças poderão gerar benefícios não apenas para os pacientes, mas também para os estudantes, uma vez que a discussão sobre dieta e exercício físico apresentará maior embasamento se os profissionais de saúde praticarem o que pretendem ensinar, simplificando a comunicação e reduzindo os fatores de risco dos próprios futuros médicos (ROBINER W, et al., 2013). 


\section{CONSIDERAÇÕES FINAIS}

As habilidades clínicas em orientações sobre modificações de estilo de vida como prevenção e manejo de pacientes com Doenças Crônicas devem fazer parte das competências almejadas para todos os estudantes do curso de medicina. De acordo com estes resultados, pode-se enfatizar que há necessidade de integração e aprimoramento curricular em habilidades clínicas direcionadas ao manejo contínuo de pacientes com fatores de risco para doenças crônicas, agregando programas de estilo de vida saudável na educação médica, uma vez que estas competências são fundamentais para a formação de médicos mais resolutivos obtendo melhores desfechos no atendimento de pacientes na atenção básica.

\section{AGRADECIMENTOS}

Aos colaboradores do Laboratório de Habilidades Clínicas da Universidade do Estado do Pará.

\section{REFERÊNCIAS}

1. ALBA LH, et al. Attitude toward preventive counseling and healthy practices among medical students at a Colombian university. Front. Med, 2015; 9(2): 251-259.

2. BERNARD, AW, et al. Medical students review of formative OSCE scores, checklists, and videos improves with student-faculty debriefing meetings. Med. Educ. Online, 2017; 22(1).

3. BOCCOLINI MM, et al. Social inequalities in limitations caused by chronic diseases and disabilities in Brazil: the 2013 National Health Survey. Cien. Saude. Colet., 2017; 22: 3537-3546.

4. CARDINAL BJ, et al. If Exercise is Medicine, Where is Exercise in Medicine? Review of U.S. Medical Education Curricula for Physical Activity-Related Content. J. Phys. Act. Health, 2015, 12 (9): 1336-1343.

5. HUGHES C, et al. Caring for the chronically ill: a clinic for final-year medical students. Medical Teacher, 2002, 24 (5): 564-566.

6. MALTA DC, et al. Mortality due to noncommunicable diseases in Brazil, 1990 to 2015, according to estimates from the Global Burden of Disease Study. Sao Paulo Med J., 2017; 135: 213-221.

7. MENDES EV. O cuidado das condições crônicas na atenção primária à saúde: o imperativo da consolidação da estratégia da saúde da família. 1. ed. Brasília: Organização Pan-Americana da Saúde, 2012: 117-233.

8. BRASIL. Ministério da Saúde. Departamento de Atenção Básica. Diretrizes para o cuidado das pessoas com doenças crônicas nas redes de atenção à saúde e nas linhas de cuidado prioritárias. Brasília:MS, 2013: 4-26.

9. EISENBERG DM, BURGESS JD. Nutrition Education in an Era of Global Obesity and Diabetes: Thinking Outside the Box. Acad. Med., 2015; 90(7): 884-860.

10. NAIR BR, FINUCANE PM. Reforming medical education to enhance the management of chronic disease. Med. J. Aust., 2003; 179(5): 257-259.

11. O'BRIEN MW, et al. Health care provider confidence and exercise prescription practices of Exercise is Medicine Canada workshop attendees. Appl. Physiol. Nutr. Metab., 2017; 42 (4): 384-390.

12. POLAK R, et al. Bridging the gap - planning Lifestyle Medicine fellowship curricula: A cross sectional study. BMC Med. Educ., 2014; 30 (14): 1-7.

13. POLAK R, et al. Medical students as health coaches: Implementation of a student-initiated Lifestyle Medicine curriculum. Isr. J. Health Policy Res., 2017; 10 (6).

14. REMUS KE, et al. A Chronic Disease Management Student-Faculty Collaborative Practice: Educating Students on Innovation in Health Care Delivery. Acad. Med., 2016; 91 (7): 967-971.

15. ROBINER W, et al. Promoting health behaviours in medical education. Clin. Teach., 2013; 10 (3): 160-164.

16. SAUCIER AN, et al. Patient perspectives of an individualized diabetes care management plan. Eur. J. Cent. Healthc., 2017; 5 (2): 213-219.

17. SIERPINA VS, et al. What Competencies Should Medical Students Attain in Nutritional Medicine. Explore, 2016; 12 (2): $146-147$. 